Article

\title{
Prediction of Equilibrium Phase, Stability and Stress-Strain Properties in Co-Cr-Fe-Ni-Al High Entropy Alloys Using Artificial Neural Networks
}

\author{
Nicolae Filipoiu ${ }^{1,2}$ and George Alexandru Nemnes ${ }^{1,2,3, *}$ \\ 1 Horia Hulubei National Institute for Physics and Nuclear Engineering, 077126 Magurele-Ilfov, Romania; \\ nicolae.filipoiu@nipne.ro \\ 2 Faculty of Physics, Materials and Devices for Electronics and Optoelectronics Research Center, \\ University of Bucharest, 077125 Magurele-Ilfov, Romania \\ 3 Research Institute of the University of Bucharest (ICUB), Mihail Kogalniceanu Blvd 36-46, \\ 050107 Bucharest, Romania \\ * Correspondence: nemnes@solid.fizica.unibuc.ro; Tel.: +40-(0)-21-457-4949/157
}

Received: 13 October 2020; Accepted: 19 November 2020; Published: 24 November 2020

check for updates

\begin{abstract}
High entropy alloys (HEAs) are still a largely unexplored class of materials with high potential for applications in various fields. Motivated by the huge number of compounds in a given HEA class, we develop machine learning techniques, in particular artificial neural networks, coupled to ab initio calculations, in order to accurately predict some basic HEA properties: equilibrium phase, cohesive energies, density of states at the Fermi level and the stress-strain relation, under conditions of isotropic deformations. Known for its high tensile ductility and fracture toughness, the Co-Cr-Fe-Ni-Al alloy has been considered as a test candidate material, particularly by adjusting the Al content. However, further enhancement of the microstructure, mechanical and thermal properties is possible by modifying also the fractions of the base alloy. Using deep neural networks, we map structural and chemical neighborhood information onto the quantities of interest. This approach offers the possibility for an efficient screening over a huge number of potential candidates, which is essential in the exploration of multi-dimensional compositional spaces.
\end{abstract}

Keywords: high entropy alloys; density functional theory; artificial neural networks

\section{Introduction}

High entropy alloys (HEAs) have gained significant interest in the past few years due to their potential use in various applications [1-3], encompassing fields like electronics, aerospace industry, hydrogen storage, coating technology, as well as base materials for components in nuclear facilities. Typically, HEAs exhibit superior strength [4], wear [5], corrosion [6,7], oxidation [8] and radiation [9] resistance, high hardness [10] and a good thermal stability [11]. The concept of HEA dates back to the seminal works of Cantor et al. [12] and Yeh et al. [13], where an exploration of the central region of multicomponent alloy phase space was first proposed and the possibility to use configurational entropy to control single-phase solid solutions versus intermetallic microstructures was indicated. The widely recognized definition of HEA assumes alloys composed of five or more elements, usually with equimolar or near-equimolar ratios. Since then, new classes of HEAs have been proposed, such a refractory HEAs [14] and HEAs with distinguished magnetic [15] and magnetocaloric [16,17] properties.

The enormous range of possible combinations poses a tough challenge from both a theoretical and experimental point of view, which makes direct exhaustive approaches rather unsuitable. Instead, the focus is on modifying and ultimately enhancing the physical properties of base alloys, e.g., CoCrFeNi alloy kernel, for adjusting the microstructure, mechanical and thermal properties. For instance, 
the CoCrFeNi alloy has been thoroughly investigated, particularly for its high tensile ductility and fracture toughness [18]. To increase the strength of the base alloy, several other CoCrFeNi-based HEAs have been considered, by including $\mathrm{Al}$ [19] and, in addition, other transition metals like $\mathrm{Ti}$ [20], Nb [21], Mn [22]. Furthermore, to diversify the HEA design, changing the composition of the base elements was suggested [18], which dramatically increases the number of potential alloy candidates.

Experimental investigations [23-25] of the $\mathrm{CoCrFeNiAl}_{x}$ alloy have previously shown that the structural configuration changes from single FCC to a duplex phase FCC/BCC, and finally reaching a single $\mathrm{BCC}$ phase as the $\mathrm{Al}$ concentration increases. This is further confirmed by theoretical stability calculations [26,27].

To handle the massive task of processing of the huge number of instances, more effective approaches are required to aid or complement traditional high throughput simulation schemes. So far, machine learning (ML) techniques have been employed for the guided exploration of phase design, using artificial neural networks (ANNs), convolutional neural networks and support vector machines [28,29], for predicting amorphous, intermetallic and solid solution character of the sample. ANNs were employed to predict the hardness using measurement records [30]. Recently, a property-oriented materials design strategy was developed using experimental data [31].

Here, we investigate HEAs based on the CoCrFeNi alloy kernel with additional Al using density functional theory (DFT) high throughput calculations, augmented by machine learning techniques, in particular, artificial neural networks (ANNs). The huge number of potential HEA candidates in a given class makes the ANN based approach an efficient tool for screening compounds targeting a given physical property. We focus here on four basic quantities, namely the total energy, the cohesive energy, the density of states at the Fermi energy and the stress induced by given strain. These are significant for phase prediction and stability and can further bring insights into magnetic and mechanical properties of HEAs. Within our approach, the ANNs are trained using the collected DFT data, using for inputs readily available structural information, i.e., the proportion and the chemical environment of each species and supercell dimensions, together with additional chemical neighborhood information.

\section{Models and Methods}

For the investigation of Co-Cr-Fe-Ni-Al HEA, we employ $a b$ initio calculations at DFT level using a supercell approach. Typical instances of FCC and BCC structures, with $2 \times 2 \times 2$ unit cells, are depicted in Figure 1. The supercells are constructed in two steps. First, the base alloy elements $(\mathrm{Co}, \mathrm{Cr}, \mathrm{Fe}, \mathrm{Ni})$ in equimolar fractions are randomly distributed, each of the species having 8 atoms (FCC) and 4 atoms (BCC). In a second step, Al is introduced substitutionally on the base elements. The supercells constructed in this way have 32 atoms (FCC) and 16 atoms (BCC) respectively. For FCC structures, a number of $n_{\mathrm{Al}}=1,2,4,6 \mathrm{Al}$ atoms are substituted for each transition metal species to yield four compounds with finite $\mathrm{Al}$ content. Correspondingly, for BCC structures, half of the $\mathrm{Al}$ atoms have been used to match the same concentration. An exception is the lowest concentration $\left(n_{\mathrm{Al}}=1\right)$, where a doubled supercell was considered in the BCC phase, i.e., with the size of 32 atoms. In order to reach the same molar fraction $x$, for both FCC and BCC, the number of $\mathrm{Al}$ atoms is always doubled in the FCC systems. This condition is later relaxed and samples with completely random atom fractions are assembled. In a typical calculation, the lattice constant is varied and the minimum of the total energy gives the equilibrium structure. The minimum total energy values are then compared for FCC and BCC structures and the equilibrium phase is identified. Due to disorder effects, several samples for the same distribution of species are considered and the averaged values are calculated. However, as is outlined in the next section, these deviations are typically not significant, as the number of atoms in the supercells is large enough to mostly cancel the disorder effects. The imposed periodicity by the supercell approach brings difficulties in describing random systems. An alternative approach is to employ the special quasi-random structures method in order to identify the best choice for the atomic configuration in small supercells [32]. However, one has to assess the computational effort needed to 
find the optimal configuration, especially since, in general, the primary advantage of the ANN based method relies on easy-to-obtain feature vectors and a relatively large volume of input data.

(a)

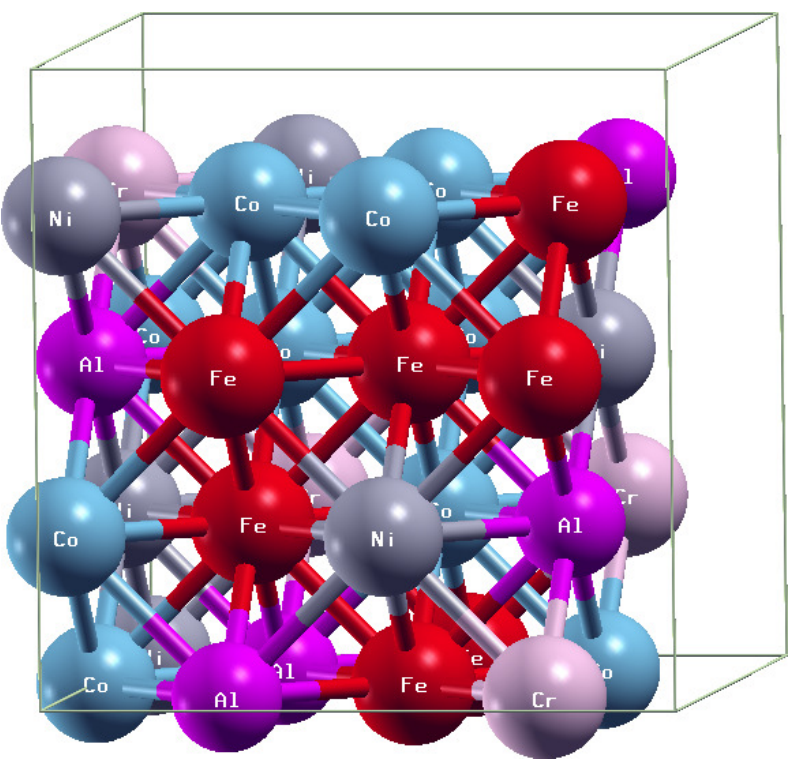

(b)

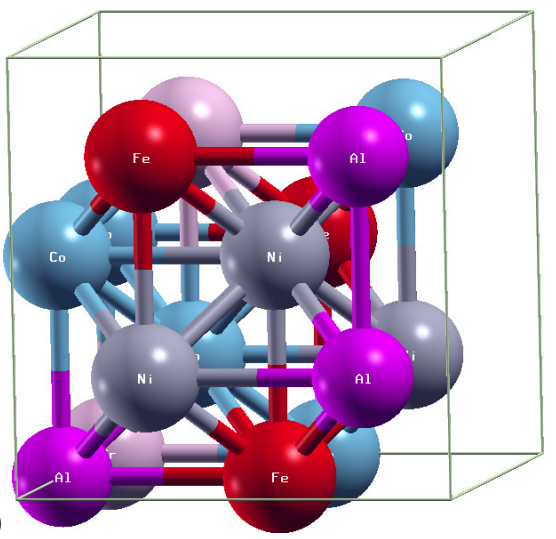

Figure 1. Typical $2 \times 2 \times 2$ supercells of Co-Cr-Fe-Ni-Al HEAs: (a) FCC structure with 32 atoms and (b) BCC structure with 16 atoms. Only the atoms belonging to one supercell (FCC/BCC) are represented.

The DFT calculations are implemented using the SIESTA package [33], which has the advantage of linear scaling of the computational time with the system size, achieved by using strictly localized atomic numeric orbitals. For the exchange-correlation potential, we employed the local density approximation (LDA) in the parametrization proposed by Ceperley and Alder [34] and norm-conserving pseudopotentials by Troullier and Martins [35]. The self-consistent solution of the Kohn-Sham equations was obtained using a double- $\zeta$ polarized basis, a mesh cutoff of 150 Ry for the real space grid and a $9 \times 9 \times 9$ Monkhorst-Pack grid was used for the k-points sampling. A broadening of $0.05 \mathrm{eV}$ is applied to the density of states (DOS).

Following DFT calculations, several quantities of interest are determined: total energy $E_{\text {tot, }}$ cohesive energy $E_{\mathrm{coh}}$, density of states at the Fermi level $D\left(E_{\mathrm{F}}\right)$ and the average normal stress component $\langle\sigma\rangle$ in the cubic systems, by pre-setting the lattice constants $\left(a_{\mathrm{FCC}}, a_{\mathrm{BCC}}\right)$. The cohesive energy is defined as the difference between the total energy of the system and the individual atomic constituents, i.e., $E_{\mathrm{coh}}=E_{\mathrm{tot}}-\sum_{i} n_{i} E_{i}$, where $n_{i}$ is the number of atoms in species $i$ and $E_{i}$ is the corresponding total energy of the isolated atoms, calculated with the same type of approximations and correlation-exchange potentials. The cohesive energy is one of the most important physical parameters in quantifying the thermal stability of materials [36], as illustrated for metallic clusters. The stress is obtained by averaging the values corresponding to the three orthogonal directions, due to an equal strain applied to each direction, i.e., $\langle\sigma\rangle=\left(\sigma_{x x}+\sigma_{y y}+\sigma_{z z}\right) / 3$. Although we consider here only isotropic deformations, the method can be employed to investigate anisotropic deformations and shear stress.

The artificial neural networks (ANNs) are implemented using TensorFlow with the Keras frontend [37,38]. One key element for the ANN accuracy is the set-up of the input feature vectors, which should include readily available information. Here, we introduce a 21-feature input vector format, with the following structure: 5 entries corresponding to the species proportions $\left(M_{i}=\mathrm{Co}\right.$, $\mathrm{Cr}, \mathrm{Fe}, \mathrm{Ni}, \mathrm{Al}), 1$ entry corresponding to the supercell dimension (lattice constant, $a_{\mathrm{FCC}}$ or $\left.a_{\mathrm{BCC}}\right)$ and 15 entries corresponding to the proportions of nearest neighbor pairs $\left(M_{i} \leftrightarrow M_{j}\right)$. This set-up of the feature vectors takes into account structural information, as well as chemical neighborhood data, which enables an efficient and an accurate prediction of the targeted quantities. A related approach 
was successfully employed for the investigation of energy gaps in hybrid graphene-hexagonal boron nitride nanoflakes $[39,40]$.

Technically, the ANNs are implemented as deep neural networks with 9 hidden layers, with $N_{\text {in }}=21$ inputs and $N_{\text {out }}=1$ output, corresponding to one of the predicted quantities. The number of neurons in the hidden layers, implemented as fully connected (dense) layers, are: $300,250,200,150,100,75,50,25,1$. All neurons, except the one in last layer use relu as activation function. The weights and biases are initialized as random uniform. Adam optimizer is employed with a learning rate of $10^{-5}$ and typical values $\beta_{1}=0.9, \beta_{2}=0.999$. The loss function is the mean squared error and accuracy is measured by the statistical coefficient of determination $R^{2}$. The DFT data are split into train, validation and test disjoint sub-sets. The ANN model is trained on a typical 5000 epochs interval, using a batch size of 100 examples.

\section{Results and Discussion}

We start our investigation by using a class of HEAs with equimolar ratios of the base elements $(\mathrm{Co}, \mathrm{Cr}, \mathrm{Fe}, \mathrm{Ni})$ and varying $\mathrm{Al}$ content, i.e., compounds of type $\mathrm{CoCrFeNiAl} x$. Equivalently, the system can be described as a pseudobinary alloy, of type $(\mathrm{CoCrFeNi})_{1-y} \mathrm{Al}_{y}$, with $y=x /(4+x)$ [26]. Two sets of supercells are assembled, one for FCC and one for BCC structural configurations, as follows: each set contains 500 samples, corresponding to 10 values for the lattice constant $a_{\mathrm{FCC}} / a_{\mathrm{BCC}}, 10$ disorder realizations and $5 \mathrm{Al}$ concentrations.

The equilibrium structural configurations are determined by varying the lattice constant and by evaluating the minimum energy, instead of performing structural relaxations. To obtain the equilibrium phase, we compare the disorder-averaged total energies in the FCC and BCC phases, $E_{\text {tot }}^{F C C}$ and $E_{\text {tot }}^{B C C}$, respectively. Figure 2a shows the total energy difference, $\Delta E_{\text {tot }}=E_{\text {tot }}^{F C C}-E_{\text {tot }}^{B C C}$. Its sign determines the equilibrium phase, $\Delta E_{\text {tot }}<0$ for FCC and $\Delta E_{\text {tot }}>0$ for BCC. A first observation is that the FCC phase is favored in the case of the base alloy, $\mathrm{CoCrFeNi}$, i.e., with no $\mathrm{Al}$ content $(x=0)$, which is consistent with reported experimental data [41,42]. As the $\mathrm{Al}$ content increases, there is an evident trend towards stabilizing the $\mathrm{BCC}$ phase. As noted before [23-25], at low $\mathrm{Al}$ fractions $(x<0.3)$, the equilibrium phase is FCC $[43,44]$, while at larger $\mathrm{Al}$ fractions $(x \approx 0.9)$, the BCC phase becomes stabilized, while mixed FCC-BCC phases are typically found for intermediate $\mathrm{Al}$ concentrations [45]. We also explore structures with high $\mathrm{Al}$ content, where the FCC phase is recovered, as shown in Figure 2. Starting from an average lattice constant of $3.42 \AA$ for the CoCrFeNi base alloy (FCC), for larger Al concentrations, the lattice constant is systematically increased and the same trend is observed for BCC configurations.

By far most of the experimental and theoretical investigations have been focused on the relatively narrow sub-set of systems with equimolar base elements. However, in general, the variation of all HEA constituents may be of interest for optimizing a certain physical property. A very recent study investigated nonequimolar $\mathrm{Co}-\mathrm{Cr}-\mathrm{Fe}-\mathrm{Ni}$-Al HEAs in comparison with the equimolar $\mathrm{CoCrFeNiAl}_{x}$ counterpart [46]. It was revealed that there is a different solubility behavior of $\mathrm{Al}$, with larger values at higher temperatures for the nonequimolar HEA, while both types of HEAs follow the same empirical phase formation rules. In particular, the FCC phase was identified for low $\mathrm{Al}$ content, followed by a mixed FCC+BCC phase for larger $\mathrm{Al}$ proportions. 

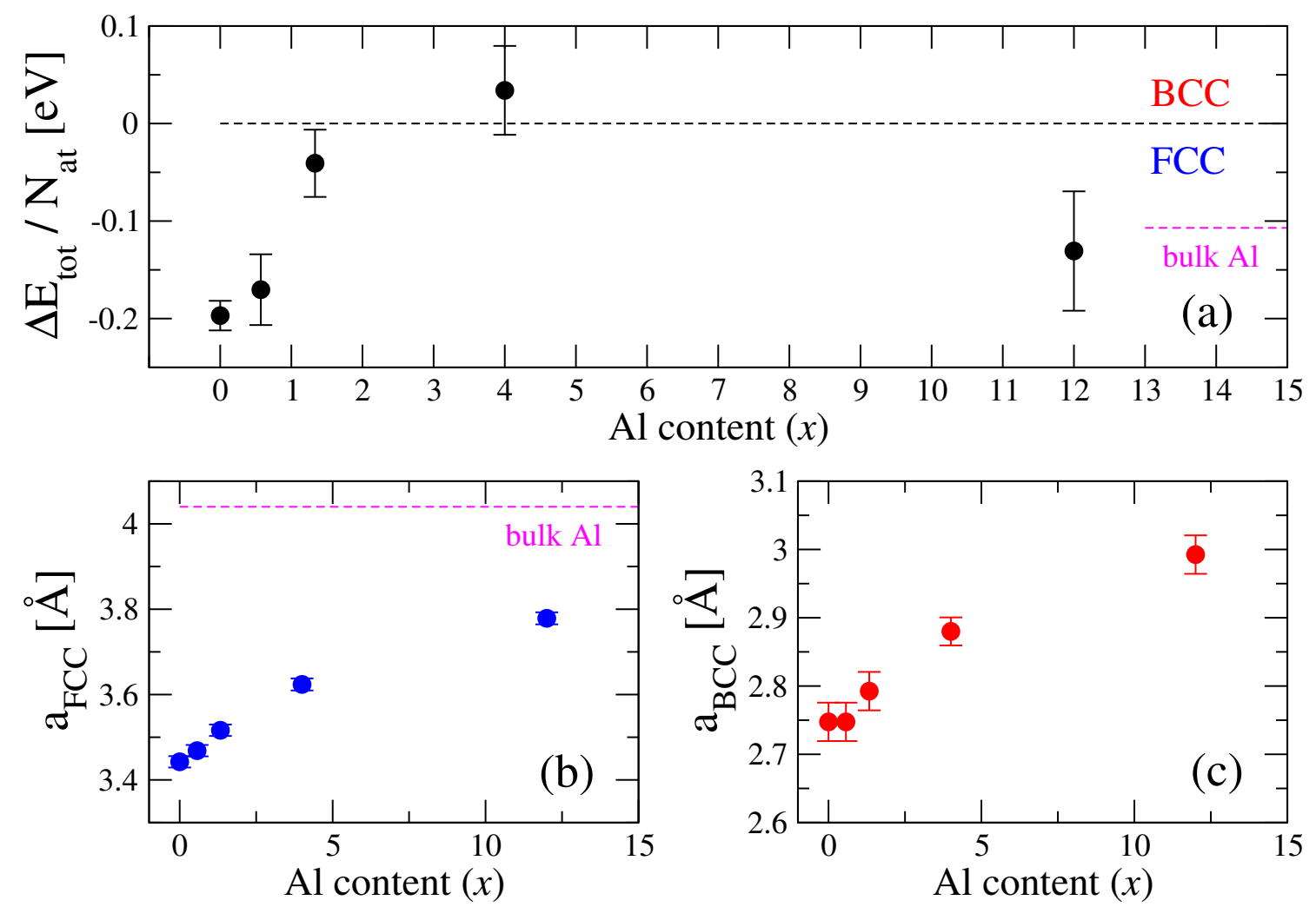

Figure 2. (a) Total energy difference between the FCC and BCC configurations per atom, $\Delta E_{\mathrm{tot}} / N_{\mathrm{at}}$ $\left(N_{\mathrm{at}}=32\right)$, as a function of $\mathrm{Al}$ content $x$, indicating the equilibrium phase. The variation of the equilibrium lattice constant with the Al content $x$ for FCC (b) and BCC (c). The experimental value for bulk $\mathrm{Al}(x \rightarrow \infty)$ is shown for reference.

Nonequimolar HEA systems require the exploration of a vast configurational state space, which cannot be performed exhaustively. In this context, we develop here machine learning techniques, based on artificial neural networks, to accurately predict key quantities necessary to determine the equilibrium phase, stability and mechanical properties.

Along these lines, we first perform DFT calculations on a set of 3000 samples with random fractions of the five elements, for each structural configuration, FCC and BCC. The two sets are each further split into 2000 samples for training the ANN, 500 for validation and 500 for test. Although there are multiple ways to train the ANN models, preliminary optimization tests indicate that an ANN architecture with 9 layers, 100 samples per batch and several other key aspects like the relu activation function, Adam optimizer provides accurate enough predictions.

It is worth highlighting that the input data are organized as feature vectors containing only directly available structural information. The 21-position feature vectors include the lattice constant, the species proportions and the proportion of nearest neighbors pairs providing additional information concerning the chemical neighborhood. The species proportions together with the lattice constant form already a highly relevant list of features. Figure 3 shows heatmap maps containing counts of samples using $E_{\text {tot }}$ vs. species proportions $\left(n_{X}\right)$ coordinates. The results are qualitatively similar for FCC and BCC, while more significant differences are visible for the five metal species, ordered in increasing atomic number. This suggests that the species proportions are good candidates for the input feature vectors. However, this limited set of features cannot accomodate situations where different configurations, with the same lattice constant and composition, can lead e.g., to different $E_{\text {tot }}$ values. To ensure a proper mapping of the input structural information, in the context of the variability induced by disorder at the same species proportions and lattice constant, the proportions of atomic pairs were added to the feature list. The feature list can be further enlarged by considering the atomic clusters of three or more atoms. Typically, as the number of possible features grows rapidly, a selection procedure 
must be enforced. As we show in the following, the selected 21-feature vector is a reasonable choice for accurate predictions of the four quantities of interest.

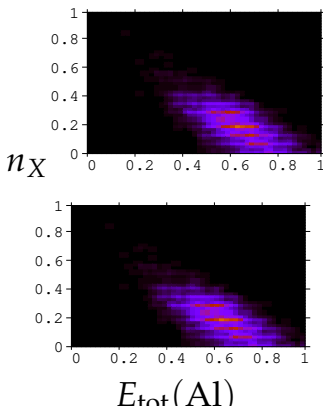

$E_{\text {tot }}(\mathrm{Al})$
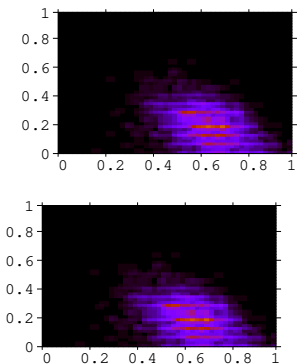

$E_{\text {tot }}(\mathrm{Cr})$
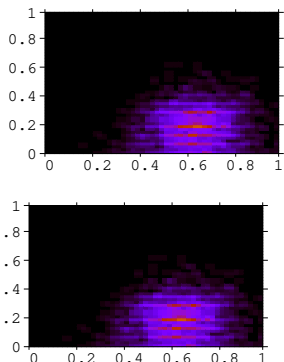

$E_{\text {tot }}(\mathrm{Fe})$
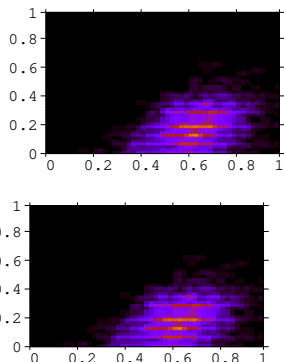

$E_{\mathrm{tot}}(\mathrm{Co})$
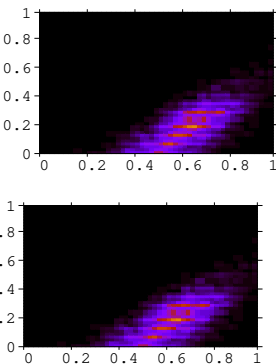

$E_{\text {tot }}(\mathrm{Ni})$

Figure 3. Heatmaps showing the number of samples in scaled $\left(E_{\mathrm{tot}}, n_{X}\right)$ coordinates, with $X=$ $\mathrm{Al}, \mathrm{Cr}, \mathrm{Fe}, \mathrm{Co}, \mathrm{Ni}$. Data corresponding to FCC (upper row) and BCC (lower row) is presented.

The validation sets provide a stopping criterion in the ANN training in order to maximize the accuracy. Afterwards, the ANN model is applied on distinct test set. Figure 4 shows the accuracy, measured by the $R^{2}$ coefficient of determination, for the quantities of interest, namely $E_{\mathrm{tot}}, E_{\mathrm{coh}}, D\left(E_{\mathrm{F}}\right)$ and $\langle\sigma\rangle$. Correspondingly, Figure 5 shows the loss functions in each case, given by the mean squared error of reference and current output values in the training process. With few exceptions, there is a very good convergence of the ANN model in less than 1000 epochs. The validation and test data closely follow the training process, which is indicative of the model consistency. The ANN models typically show improved performance for the BCC structural configurations, as compared to the FCC case. This may be due to the lower complexity of the BCC supercells with only half of the number of atoms in the FCC supercells. Among the monitored output parameters, $E_{\text {tot }}$ was most accurately predicted with a rather quick convergence of the ANN model, followed by $D\left(E_{\mathrm{F}}\right)$ and $\langle\sigma\rangle$. For these three parameters, the obtained accuracies typically exceed 99\%. The cohesive energies are mapped with less precision and an overtraining effect is evidenced in Figures $4 \mathrm{~b}$ and $5 \mathrm{~b}$ : in the training process, $R^{2}$ increases (the loss function decreases) monotonically in the FCC case, while the validation and test data present a maximum for $R^{2}$ (minimum for MSE). In this context, the maximum $R^{2}$ for the validation set, of $\sim 75 \%$, indicates the optimum training point at $\sim 2000$ epochs. The accuracy for the test set also presents a maximum near this point, with a value for $R^{2}$ of only $68 \%$. However, for the BCC case, the overtraining effect is much less prominent and the convergence and accuracy are significantly improved.

Furthermore, we investigate the accuracy of phase prediction by using a singleANN for a set samples randomly distributed between FCC and BCC configurations. As a test case, the total energies of 250 structures with the same composition, but randomly chosen lattice constants, have been determined by DFT calculations. The HEA composition was set by equimolar $\mathrm{Co}, \mathrm{Cr}, \mathrm{Fe}, \mathrm{Ni}$, with an Al content corresponding to $x=4 / 7$, i.e., one $\mathrm{Al}$ atom to seven atoms of each transition metal species. In order to have a direct comparison, the BCC cell was doubled along the z-direction, yielding an equal number of atoms as in the FCC supercell.

The predicted values of $E_{\text {tot }}$ are presented in Figure 6, in comparison with the reference values and the equilibrium phase can be determined from the minimum energy. In this instance, a slightly lower $E_{\text {tot }}$ was found, compared FCC to BCC, which is typical for this Al proportion. The obtained accuracy for the test set is $R^{2}=99.2 \%$, which is high enough to distinguish between the two phases. The total energy and the lattice constants have been linearly transformed, so that the new corresponding parameters $\tilde{E}_{\text {tot }}$ and $\tilde{a}$ are found in $(0,1)$. This procedure ensures a smooth convergence of the ANN model in the training process. 

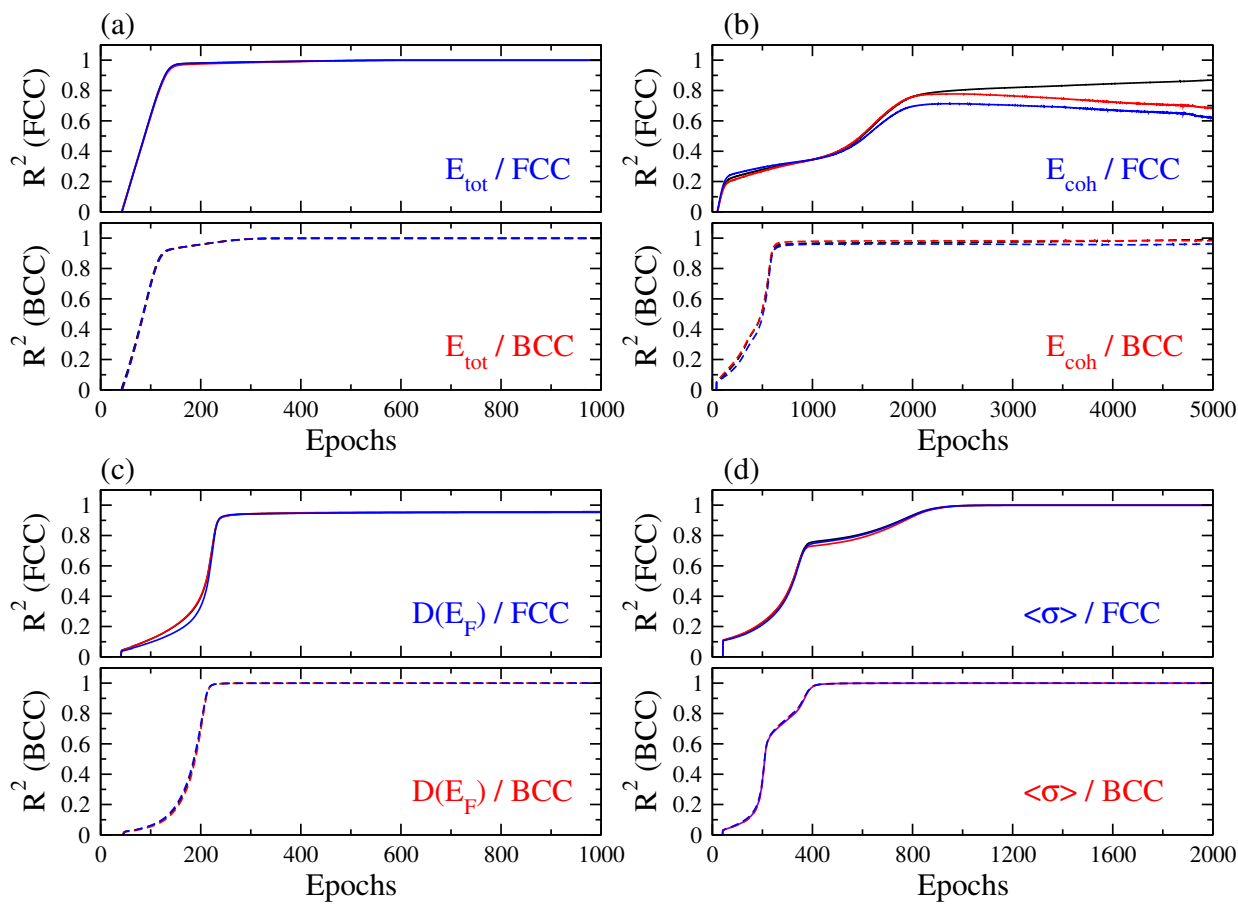

Figure 4. The accuracies of the ANN models, measured by $R^{2}$, during training (black), validation (red) and test (blue), for: (a) $E_{\text {tot }},(\mathbf{b}) E_{\text {coh }},(\mathbf{c}) D\left(E_{\mathrm{F}}\right)$ and (d) $\langle\sigma\rangle$. The data are presented for FCC (solid) and BCC (dashed) structural configurations.
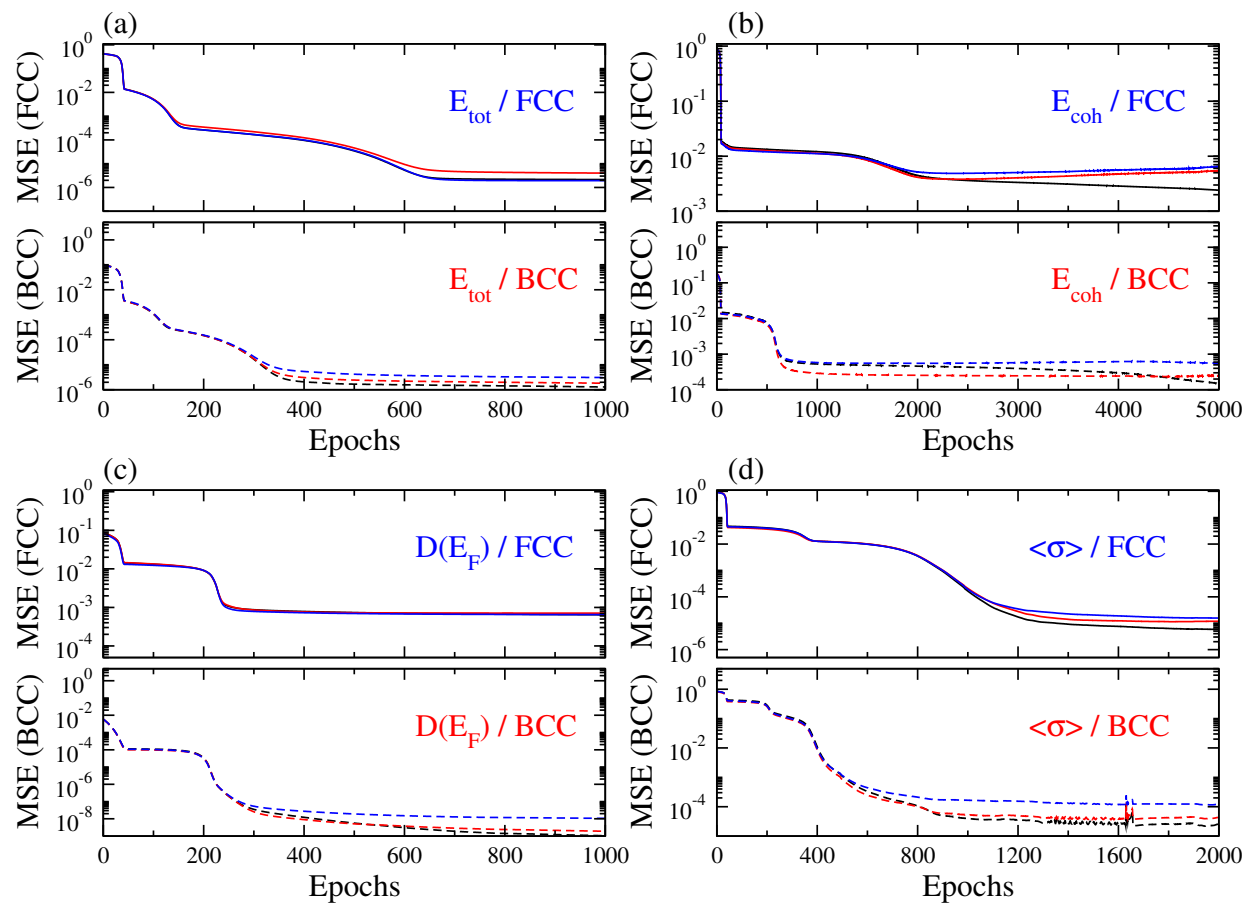

Figure 5. The loss functions (mean squared error) for training, validation and test, in correspondence with $r^{2}$ coefficients presented in Figure $4 \mathrm{a}-\mathrm{d}$. Overtraining effects are visible in the (b) sub-plot, mapping cohesive energies.

It is worth noting that the FCC/BCC overlap region, outlined by dashed lines, is also well mapped, proving that the choice of the feature vector can discriminate between different structural configurations. Depending on the candidate HEA class, the ML methods introduced here can be easily adapted to include other structural phases of interest, like the hexagonal closely packed phase. 
Moreover, although we only investigated here some basic quantities, which can be determined directly from $a b$ initio calculations, other related properties can be further derived and predicted. In particular, by including anisotropic and shear stress, a more detailed description of mechanical properties can be performed, which is of practical interest. Moreover, our approach is general enough to accommodate output quantities which can be obtained with theories beyond DFT and can be extended to include external conditions like temperature and pressure.

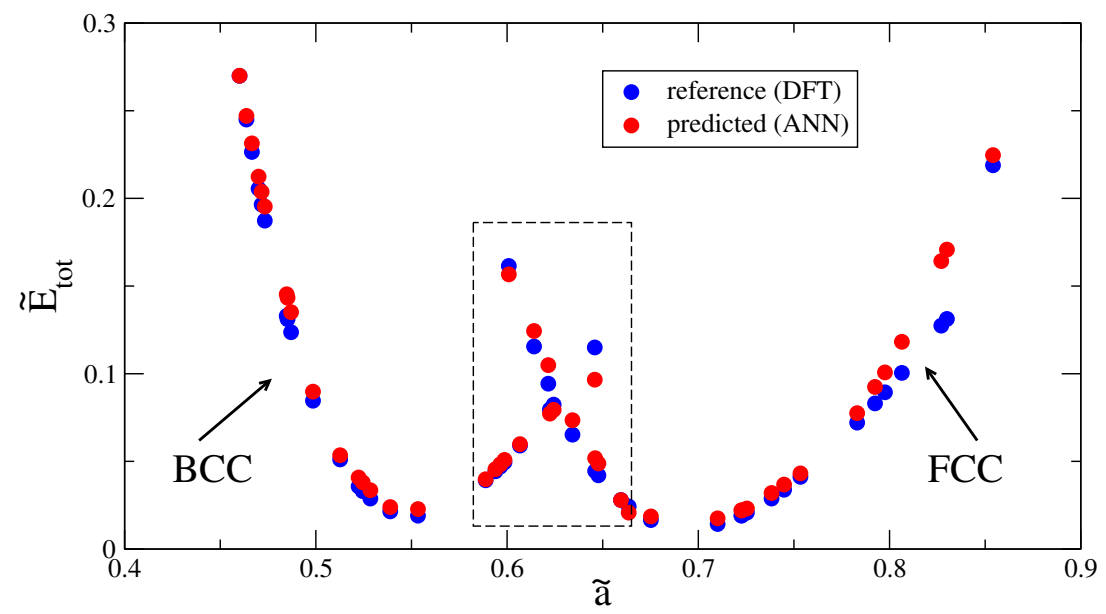

Figure 6. Rescaled total energy vs. lattice constant for a pre-set composition, with 32 atoms in the supercell, $\mathrm{Co}(7)-\mathrm{Cr}(7)-\mathrm{Fe}(7)-\mathrm{Ni}(7)-\mathrm{Al}(4)$, and random choice of the lattice constant and structural configuration (FCC and BCC). The region enclosed by the dashed lines marks the crossing of the FCC and BCC curves.

\section{Conclusions}

Machine learning techniques have been developed and employed for the investigation of Co-Cr-Fe-Ni-Al HEAs, mapping DFT results. Using deep neural networks, several quantities of interest have been successfully predicted, such as total and cohesive energies, density of states at the Fermi level and the stress induced by a given strain. These quantities are essential to determine the equilibrium phase and stability, and for assessing the electronic and mechanical properties. The input feature vectors are constructed using directly available information, consisting of HEA composition, supercell dimensions and chemical neighborhood descriptors. Furthermore, we showed that this approach is able to distinguish between different structural phases. Although high throughput DFT calculations are widely used for the exploration of multi-component configurational state space, the ML techniques provide a significant step forward by efficiently screening a huge number of candidates, HEAs being in particular suited for this type of approach.

Author Contributions: All authors contributed equally. All authors have read and agreed to the published version of the manuscript.

Funding: The authors acknowledge the financial support from the Ministry of Education and Scientific Research under project PN 19060205/2020 and European Regional Development Fund through project CeCBID-EOSC (POC/397/1/1-124405).

Conflicts of Interest: The authors declare no conflict of interest.

\section{References}

1. Gorsse, S.; Couzinié, J.P.; Miracle, D.B. From high-entropy alloys to complex concentrated alloys. Comptes Rendus Phys. 2018, 19, 721-736. [CrossRef]

2. Chen, J.; Zhou, X.; Wang, W.; Liu, B.; Lv, Y.; Yang, W.; Xu, D.; Liu, Y. A review on fundamental of high entropy alloys with promising high-temperature properties. J. Alloys Compd. 2018, 760, 15-30. [CrossRef]

3. George, E.P.; Raabe, D.; Ritchie, R.O. High-entropy alloys. Nat. Rev. Mater. 2019, 4, 515-534. [CrossRef] 
4. Qiu, S.; Miao, N.; Zhou, J.; Guo, Z.; Sun, Z. Strengthening mechanism of aluminum on elastic properties of NbVTiZr high-entropy alloys. Intermetallics 2018, 92, 7-14. [CrossRef]

5. Wu, J.M.; Lin, S.J.; Yeh, J.W.; Chen, S.K.; Huang, Y.S.; Chen, H.C. Adhesive wear behavior of AlxCoCrCuFeNi high-entropy alloys as a function of aluminum content. Wear 2006, 261, 513-519. [CrossRef]

6. Chuang, M.H.; Tsai, M.H.; Wang, W.R.; Lin, S.J.; Yeh, J.W. Microstructure and wear behavior of AlxCo1.5CrFeNi1.5Tiy high-entropy alloys. Acta Mater. 2011, 59, 6308-6317. [CrossRef]

7. Qiu, Y.; Thomas, S.; Gibson, M.A.; Fraser, H.L.; Birbilis, N. Corrosion of high entropy alloys. Npj Mater. Degrad. 2017, 1, 15. [CrossRef]

8. Huang, P.K.; Yeh, J.W.; Shun, T.T.; Chen, S.K. Multi-Principal-Element Alloys with Improved Oxidation and Wear Resistance for Thermal Spray Coating. Adv. Eng. Mater. 2004, 6, 74-78. [CrossRef]

9. Kumar, N.K.; Li, C.; Leonard, K.; Bei, H.; Zinkle, S. Microstructural stability and mechanical behavior of FeNiMnCr high entropy alloy under ion irradiation. Acta Mater. 2016, 113, 230-244. [CrossRef]

10. Yu, P.; Zhang, L.; Cheng, H.; Zhang, H.; Ma, M.; Li, Y.; Li, G.; Liaw, P.; Liu, R. The high-entropy alloys with high hardness and soft magnetic property prepared by mechanical alloying and high-pressure sintering. Intermetallics 2016, 70, 82-87. [CrossRef]

11. Sriharitha, R.; Murty, B.; Kottada, R.S. Alloying, thermal stability and strengthening in spark plasma sintered AlxCoCrCuFeNi high entropy alloys. J. Alloys Compd. 2014, 583, 419-426. [CrossRef]

12. Cantor, B.; Chang, I.; Knight, P.; Vincent, A. Microstructural development in equiatomic multicomponent alloys. Mater. Sci. Eng. A 2004, 375-377, 213-218. [CrossRef]

13. Yeh, J.W.; Chen, S.K.; Lin, S.J.; Gan, J.Y.; Chin, T.S.; Shun, T.T.; Tsau, C.H.; Chang, S.Y. Nanostructured High-Entropy Alloys with Multiple Principal Elements: Novel Alloy Design Concepts and Outcomes. Adv. Eng. Mater. 2004, 6, 299-303. [CrossRef]

14. Senkov, O.; Wilks, G.; Miracle, D.; Chuang, C.; Liaw, P. Refractory high-entropy alloys. Intermetallics 2010, 18, 1758-1765. [CrossRef]

15. Mishra, R.K.; Shahi, R.R. Phase evolution and magnetic characteristics of TiFeNiCr and TiFeNiCrM (M=Mn, Co) high entropy alloys. J. Magn. Magn. Mater. 2017, 442, 218-223. [CrossRef]

16. Yuan, Y.; Wu, Y.; Tong, X.; Zhang, H.; Wang, H.; Liu, X.; Ma, L.; Suo, H.; Lu, Z. Rare-earth high-entropy alloys with giant magnetocaloric effect. Acta Mater. 2017, 125, 481-489. [CrossRef]

17. Sarlar, K.; Tekgül, A.; Kucuk, I. Magnetocaloric properties in a FeNiGaMnSi high entropy alloy. Curr. Appl. Phys. 2020, 20, 18-22. [CrossRef]

18. He, F.; Wang, Z.; Wu, Q.; Niu, S.; Li, J.; Wang, J.; Liu, C. Solid solution island of the Co-Cr-Fe-Ni high entropy alloy system. Scr. Mater. 2017, 131, 42-46. [CrossRef]

19. Ma, S.; Zhang, S.; Qiao, J.; Wang, Z.; Gao, M.; Jiao, Z.; Yang, H.; Zhang, Y. Superior high tensile elongation of a single-crystal CoCrFeNiAl0.3 high-entropy alloy by Bridgman solidification. Intermetallics 2014, 54, 104-109. [CrossRef]

20. He, J.; Wang, H.; Huang, H.; Xu, X.; Chen, M.; Wu, Y.; Liu, X.; Nieh, T.; An, K.; Lu, Z. A precipitationhardened high-entropy alloy with outstanding tensile properties. Acta Mater. 2016, 102, 187-196. [CrossRef]

21. Ma, S.; Zhang, Y. Effect of $\mathrm{Nb}$ addition on the microstructure and properties of AlCoCrFeNi high-entropy alloy. Mater. Sci. Eng. A 2012, 532, 480-486. [CrossRef]

22. Prasad, H.; Singh, S.; Panigrahi, B.B. Mechanical activated synthesis of alumina dispersed FeNiCoCrAlMn high entropy alloy. J. Alloys Compd. 2017, 692, 720-726. [CrossRef]

23. Chou, H.P.; Chang, Y.S.; Chen, S.K.; Yeh, J.W. Microstructure, thermophysical and electrical properties in AlxCoCrFeNi $(0 \leq x \leq 2)$ high-entropy alloys. Mater. Sci. Eng. B 2009, 163, 184-189. [CrossRef]

24. Kao, Y.F.; Chen, T.J.; Chen, S.K.; Yeh, J.W. Microstructure and mechanical property of as-cast, -homogenized, and -deformed AlxCoCrFeNi $(0 \leq \mathrm{x} \leq 2)$ high-entropy alloys. J. Alloys Compd. 2009, 488, 57-64. [CrossRef]

25. Wang, W.R.; Wang, W.L.; Wang, S.C.; Tsai, Y.C.; Lai, C.H.; Yeh, J.W. Effects of Al addition on the microstructure and mechanical property of AlxCoCrFeNi high-entropy alloys. Intermetallics 2012, 26, 44-51. [CrossRef]

26. Tian, F.; Delczeg, L.; Chen, N.; Varga, L.K.; Shen, J.; Vitos, L. Structural stability of NiCoFeCrAl $x$ high-entropy alloy from ab initio theory. Phys. Rev. B 2013, 88, 085128. [CrossRef]

27. Widom, M. Modeling the structure and thermodynamics of high-entropy alloys. J. Mater. Res. 2018, 33, 2881-2898. [CrossRef]

28. Zhou, Z.; Zhou, Y.; He, Q.; Ding, Z.; Li, F.; Yang, Y. Machine learning guided appraisal and exploration of phase design for high entropy alloys. Npj Comput. Mater. 2019, 5, 128. [CrossRef] 
29. Huang, W.; Martin, P.; Zhuang, H.L. Machine-learning phase prediction of high-entropy alloys. Acta Mater. 2019, 169, 225-236. [CrossRef]

30. Chang, Y.J.; Jui, C.Y.; Lee, W.J.; Yeh, A.C. Prediction of the Composition and Hardness of High-Entropy Alloys by Machine Learning. JOM 2019, 71, 3433-3442. [CrossRef]

31. Wen, C.; Zhang, Y.; Wang, C.; Xue, D.; Bai, Y.; Antonov, S.; Dai, L.; Lookman, T.; Su, Y. Machine learning assisted design of high entropy alloys with desired property. Acta Mater. 2019, 170, 109-117. [CrossRef]

32. Gao, M.C.; Niu, C.; Jiang, C.; Irving, D.L. Applications of Special Quasi-random Structures to High-Entropy Alloys. In High-Entropy Alloys: Fundamentals and Applications; Gao, M.C., Yeh, J.W., Liaw, P.K., Zhang, Y., Eds.; Springer International Publishing: Cham, Switzerland, 2016; pp. 333-368.

33. Soler, J.M.; Artacho, E.; Gale, J.D.; Garcia, A.; Junquera, J.; Ordejon, P.; Sanchez-Portal, D. The SIESTA method for ab initio order- N materials simulation. J. Phys. Condens. Matter 2002, 14, 2745. [CrossRef]

34. Ceperley, D.M.; Alder, B.J. Ground State of the Electron Gas by a Stochastic Method. Phys. Rev. Lett. 1980, 45, 566-569. [CrossRef]

35. Troullier, N.; Martins, J.L. Efficient pseudopotentials for plane-wave calculations. Phys. Rev. B 1991, 43, 1993-2006. [CrossRef] [PubMed]

36. Yang, C.C.; Li, S. Cohesive Energy: The Intrinsic Dominant of Thermal Stability and Structural Evolution in Sn from Size Scales of Bulk to Dimer. J. Phys. Chem. C 2009, 113, 14207-14212. [CrossRef]

37. TensorFlow. Available online: https://www.tensorflow.org/ (accessed on 1 June 2020).

38. Keras. Available online: https:// keras.io/(accessed on 1 June 2020).

39. Nemnes, G.A.; Mitran, T.L.; Manolescu, A. Gap prediction in hybrid graphene-hexagonal boron nitride nanoflakes using artificial neural networks. J. Nanometer. 2019, 2019, 6960787.

40. Mitran, T.; Nemnes, G. Theory and Simulation in Physics for Materials Applications. In Prediction of Energy Gaps in Graphene-Hexagonal Boron Nitride Nanoflakes Using Artificial Neural Networks; Springer International Publishing: Berlin, Germany, 2020; pp. 197-209

41. Zaddach, A.J.; Niu, C.; Koch, C.C.; Irving, D.L. Mechanical Properties and Stacking Fault Energies of $\mathrm{NiFeCrCoMn} \mathrm{High-Entropy} \mathrm{Alloy.} \mathrm{JOM} \mathrm{2013,} \mathrm{65,} \mathrm{1780-1789.} \mathrm{[CrossRef]}$

42. Tang, Z.; Senkov, O.N.; Parish, C.M.; Zhang, C.; Zhang, F.; Santodonato, L.J.; Wang, G.; Zhao, G.; Yang, F.; Liaw, P.K. Tensile ductility of an AlCoCrFeNi multi-phase high-entropy alloy through hot isostatic pressing (HIP) and homogenization. Mater. Sci. Eng. A 2015, 647, 229-240. [CrossRef]

43. Li, D.; Li, C.; Feng, T.; Zhang, Y.; Sha, G.; Lewandowski, J.J.; Liaw, P.K.; Zhang, Y. High-entropy Al0.3CoCrFeNi alloy fibers with high tensile strength and ductility at ambient and cryogenic temperatures. Acta Mater. 2017, 123, 285-294. [CrossRef]

44. Wu, Z.; Bei, H.; Otto, F.; Pharr, G.; George, E. Recovery, recrystallization, grain growth and phase stability of a family of FCC-structured multi-component equiatomic solid solution alloys. Intermetallics 2014, 46, 131-140. [CrossRef]

45. Li, Q.; Zhang, T.; Qiao, J.; Ma, S.; Zhao, D.; Lu, P.; Wang, Z. Mechanical properties and deformation behavior of dual-phase Al0.6CoCrFeNi high-entropy alloys with heterogeneous structure at room and cryogenic temperatures. J. Alloys Compd. 2020, 816, 152663. [CrossRef]

46. Mehta, A.; Sohn, Y. Interdiffusion, Solubility Limit, and Role of Entropy in FCC Al-Co-Cr-Fe-Ni Alloys. Metall. Mater. Trans. A 2020, 51, 3142-3153. [CrossRef]

Publisher's Note: MDPI stays neutral with regard to jurisdictional claims in published maps and institutional affiliations.

(C) 2020 by the authors. Licensee MDPI, Basel, Switzerland. This article is an open access article distributed under the terms and conditions of the Creative Commons Attribution (CC BY) license (http:/ / creativecommons.org/licenses/by/4.0/). 